\title{
Supplementary material to 'The impact of differences in large-scale circulation output from climate models on the regional modeling of ozone and PM'
}

\section{Statistical evaluation}

Table S1a. Annual average behavior of temperatures, 1989-2009. Standard deviations represent the day to day variability

\begin{tabular}{|c|c|c|c|c|c|c|c|c|c|c|c|c|}
\hline \multirow[t]{2}{*}{ station } & \multicolumn{4}{|c|}{ RLE_ERA } & \multicolumn{4}{|c|}{ RLE_ECHAM } & \multicolumn{4}{|c|}{ RLE_MIROC } \\
\hline & $\# \mathrm{~T}>25$ & $\# \mathrm{~T}<5$ & $\mathrm{~T}_{\max }$ & std & $\# \mathrm{~T}>25 \mathrm{C}$ & $\# \mathrm{~T}<5 \mathrm{C}$ & $\mathrm{T}_{\max }$ & Std & $\# \mathrm{~T}>25$ & $\# \mathrm{~T}<5$ & $\mathrm{~T}_{\max }$ & std \\
\hline Vredepeel & 28 & 54 & 13.63 & 7.91 & 16 & 46 & 13.12 & 7.03 & 30 & 44 & 14.12 & 7.72 \\
\hline Sniezka & 30 & 105 & 11.89 & 9.43 & 17 & 96 & 11.55 & 8.54 & 29 & 101 & 12.17 & 9.12 \\
\hline Melpitz & 37 & 81 & 13.13 & 9.09 & 20 & 73 & 12.62 & 8.2 & 39 & 74 & 13.59 & 8.89 \\
\hline Paris & 37 & 41 & 14.63 & 7.88 & 25 & 35 & 14.05 & 7.07 & 29 & 33 & 14.57 & 7.33 \\
\hline Barcelona & 126 & 1 & 21.37 & 8.46 & 124 & 1 & 21.20 & 8.21 & 99 & 1 & 20.08 & 6.99 \\
\hline Madrid & 109 & 6 & 19.21 & 9.30 & 105 & 7 & 18.57 & 9.35 & 76 & 4 & 17.43 & 7.86 \\
\hline Montelibretti & 63 & 0 & 18.27 & 5.90 & 19 & 0 & 17.19 & 5.00 & 55 & 0 & 18.24 & 5.49 \\
\hline
\end{tabular}

Table 1b. Annual average behavior of rain 1989-2009. Wet days are days with more than $0.5 \mathrm{~mm}$ rain.

\begin{tabular}{|l|rr|rr|rr|}
\hline station & RLE_ERA & & RLE_ECHAM & RLE_MIROC & \\
\hline & wet days & $\begin{array}{r}\text { yearly } \\
\text { rain (mm) }\end{array}$ & wet days & $\begin{array}{r}\text { yearly rain } \\
(\mathrm{mm})\end{array}$ & wet days & $\begin{array}{r}\text { yearly } \\
\text { rain (mm) }\end{array}$ \\
\hline Vredepeel & 196 & 748.25 & 227 & 956.3 & 199 & 751.9 \\
Sniezka & 184 & 653.35 & 212 & 803.0 & 198 & 708.1 \\
Melpitz DE44 & 168 & 532.9 & 194 & 668.0 & 175 & 584.0 \\
Paris & 179 & 689.9 & 212 & 872.4 & 201 & 759.2 \\
Barcelona & 71 & 335.8 & 80 & 383.2 & 93 & 452.6 \\
Madrid & 93 & 430.7 & 124 & 675.3 & 116 & 543.9 \\
Montelibretti & 98 & 481.8 & 109 & 595.0 & 137 & 803.0 \\
\hline
\end{tabular}

Table S1c. Annual averages wind speed 1989-2009

\begin{tabular}{|l|rr|rr|rr|r|}
\hline station & \multicolumn{2}{|l|}{ RLE_ERA } & \multicolumn{2}{l|}{ RLE_ECHAM } & \multicolumn{2}{l|}{ RLE_MIROC } \\
\hline & \#v $<2 \mathrm{~m} / \mathrm{s}$ & \multicolumn{1}{l|}{ average $\mathrm{v}$} & \#v $<2 \mathrm{~m} / \mathrm{s}$ & average $\mathrm{n}$ & \#v $<2 \mathrm{~m} / \mathrm{s}$ & average $\mathrm{v}$ \\
\hline Vredepeel & 40 & 4.0 & 32 & 4.30 & 44 & 3.85 \\
Sniezka & 55 & 3.2 & 48 & 3.40 & 66 & 3.05 \\
Melpitz DE44 & 34 & 3.99 & 28 & 4.24 & 41 & 3.75 \\
Paris & 26 & 3.87 & 23 & 4.16 & 31 & 3.83 \\
Barcelona & 123 & 2.84 & 101 & 3.14 & 125 & 2.92 \\
Madrid & 64 & 3.04 & 52 & 3.41 & 67 & 3.14 \\
Montelibretti & 34 & 3.86 & 30 & 4.35 & 40 & 4.01 \\
\hline
\end{tabular}

TableS 2a. Average behavior of temperature, 2041-2060

\begin{tabular}{|l|lrl|l|r|r|r|r|r|}
\hline station & \multicolumn{3}{|l|}{ RLE_ECHAM } & \multicolumn{3}{l|}{ RLE_MIROC } \\
\hline & \# T>25 C & \# T<5 C & $\mathrm{T}_{\max }$ & std & \# T >25 C & \# T<5 C & $\mathrm{T}_{\max }$ & std \\
\hline Vredepeel & 25 & 33 & 14.26 & 7.08 & 55 & 21 & 16.28 & 7.66 \\
Sniezka & 26 & 76 & 12.78 & 8.37 & 53 & 65 & 14.44 & 8.98 \\
Melpitz & 27 & 53 & 13.77 & 7.96 & 65 & 42 & 15.69 & 8.72 \\
Paris & 38 & 21 & 15.23 & 7.30 & 65 & 12 & 17.02 & 7.46 \\
Barcelona & 148 & 0 & 22.92 & 8.63 & 145 & 0 & 23.16 & 7.53
\end{tabular}




\begin{tabular}{|l|rrrr|rrrr|} 
Madrid & 129 & 2 & 20.72 & 10.01 & 123 & 0 & 20.97 & 8.79 \\
Montelibretti & 59 & 0 & 18.52 & 5.29 & 102 & 0 & 20.79 & 5.76 \\
\hline
\end{tabular}

Table S2b. Averages rain 2041-2060. Wet days are days with more than $0.5 \mathrm{~mm}$ rain

\begin{tabular}{|c|c|c|c|c|c|}
\hline station & RLE_ECHAM & & RLE_MIRC & & \\
\hline & wet days & $\begin{array}{l}\text { yearly rain } \\
(\mathrm{mm})\end{array}$ & wet days & $\begin{array}{l}\text { yearly } \\
\text { rain }(\mathrm{mm})\end{array}$ & \\
\hline Vredepeel & 228 & 992.8 & 199 & & 832.2 \\
\hline Sniezka & 219 & 868.7 & 202 & & 770.2 \\
\hline Melpitz & 200 & 744.6 & 177 & & 635.1 \\
\hline Paris & 210 & 887.9 & 193 & & 788.4 \\
\hline Barcelona & 74 & 335.8 & 87 & & 394.2 \\
\hline Madrid & 104 & 616.9 & 97 & & 463.6 \\
\hline Montelibretti & 103 & 609.6 & 129 & & 759.2 \\
\hline
\end{tabular}

Table S2c. Averages wind speed 2041-2060.

\begin{tabular}{|l|rr|r|r|}
\hline station & \multicolumn{2}{|l|}{ RLE_ECHAM } & \multicolumn{2}{|c|}{ RLE_MIROC } \\
\hline & \#v<2m/s & average v & \#v<2m/s & average v \\
\hline Vredepeel & 31 & 4.29 & 50 & 3.77 \\
Sniezka & 47 & 3.39 & 68 & 3.00 \\
Melpitz & 31 & 4.18 & 47 & 3.66 \\
Paris & 23 & 4.16 & 34 & 3.72 \\
Barcelona & 99 & 3.16 & 123 & 2.89 \\
Madrid & 54 & 3.38 & 61 & 3.09 \\
Montelibretti & 26 & 4.35 & 41 & 3.99 \\
\hline
\end{tabular}

Table S3. Summer average daily maximum O3 concentrations and annual average PM10 concentrations, present-day and future period. Standard deviations represent the day to day variability.

\begin{tabular}{|l|r|r|r|r|}
\hline RLE_ERA (1989-2009) & & & & \\
\hline station & \multicolumn{1}{|c|}{$\mathrm{O}_{3 \max }$} & $\mathrm{O}_{3 \max }$ & PM10 & PM10 \\
& average & stdev & average & stdev \\
\hline Vredepeel & 100.26 & 27.69 & 13.59 & 6.27 \\
Sniezka & 108.23 & 18.22 & 8.74 & 4.25 \\
Melpitz & 107.11 & 20.34 & 8.65 & 4.28 \\
Paris & 99.36 & 26.43 & 36.30 & 20.93 \\
Barcelona & 118.00 & 19.19 & 8.89 & 4.80 \\
Madrid & 97.91 & 14.75 & 17.80 & 7.39
\end{tabular}




\begin{tabular}{|l|r|l|l|l|l|l|l|l|l|r|}
\hline RLE_ECHAM & \multicolumn{2}{|l|}{$(1989-2009)$} & \multicolumn{2}{l|}{$(2041-2060)$} & \multicolumn{3}{l|}{ Relative change \% } \\
\hline station & \multicolumn{1}{|l|}{$\mathrm{O}_{3 \max }$} & $\mathrm{O}_{3 \max }$ & PM10 & PM10 & $\mathrm{O}_{3 \max }$ & $\mathrm{O}_{3 \max }$ & PM10 & PM10 & $\mathrm{O}_{3}$ & PM10 \\
& average & stdev & average & stdev & average & stdev & average & stdev & & \\
\hline Vredepeel & 90.53 & 23.63 & 12.58 & 5.90 & 93.06 & 26.95 & 12.64 & 5.97 & +2.9 & +0.5 \\
Sniezka & 102.28 & 17.31 & 8.15 & 4.05 & 105.58 & 20.13 & 7.81 & 3.88 & +3.2 & -4.2 \\
Melpitz & 99.95 & 19.30 & 8.21 & 4.12 & 102.69 & 21.28 & 7.99 & 3.98 & +2.7 & -2.7 \\
Paris & 90.59 & 23.63 & 32.33 & 20.05 & 96.16 & 27.10 & 31.67 & 19.57 & +6.1 & -2.0 \\
Barcelona & 113.09 & 19.11 & 7.56 & 4.44 & 118.76 & 21.24 & 7.76 & 4.53 & +5.0 & +2.6 \\
Madrid & 98.46 & 14.92 & 15.32 & 7.36 & 102.39 & 15.97 & 15.68 & 7.51 & +4.0 & +2.3 \\
Montelibretti & 120.19 & 16.75 & 10.05 & 4.04 & 124.30 & 18.55 & 10.01 & 4.11 & +3.4 & -0.4 \\
\hline
\end{tabular}

\begin{tabular}{|l|l|l|l|l|l|l|l|l|l|r|}
\hline RLE_MIROC & \multicolumn{9}{|l}{$(1989-2009)$} & \multicolumn{2}{l|}{$(2041-2060)$} & \multicolumn{2}{l|}{ Relative change \% } \\
\hline station & $\mathrm{O}_{3 \max }$ & $\mathrm{O}_{3 \max }$ & PM10 & PM10 & $\mathrm{O}_{3 \max }$ & $\mathrm{O}_{3 \max }$ & PM10 & PM10 & $\mathrm{O}_{3}$ & \multicolumn{1}{l|}{ PM10 } \\
& average & stdev & average & stdev & average & stdev & average & stdev & & \\
\hline Vredepeel & 106.77 & 25.87 & 13.83 & 6.38 & 114.94 & 29.29 & 14.47 & 7.18 & +7.7 & +4.6 \\
Sniezka & 109.93 & 15.93 & 8.57 & 4.17 & 116.11 & 17.67 & 8.40 & 4.30 & +5.6 & -2.0 \\
Melpitz & 111.02 & 18.55 & 8.50 & 4.32 & 116.80 & 20.49 & 8.52 & 4.60 & +5.2 & +0.2 \\
Paris & 105.27 & 26.93 & 35.63 & 20.42 & 112.56 & 30.16 & 36.35 & 19.69 & +6.9 & +2.0 \\
Barcelona & 114.51 & 17.78 & 8.04 & 4.50 & 122.14 & 18.86 & 8.15 & 4.56 & +6.7 & +0.1 \\
Madrid & 96.16 & 14.57 & 17.30 & 7.50 & 100.75 & 16.05 & 17.34 & 7.39 & +4.8 & +0.2 \\
Montelibretti & 122.61 & 16.90 & 10.08 & 4.17 & 129.75 & 11.94 & 10.25 & 4.32 & +5.8 & +1.6 \\
\hline
\end{tabular}



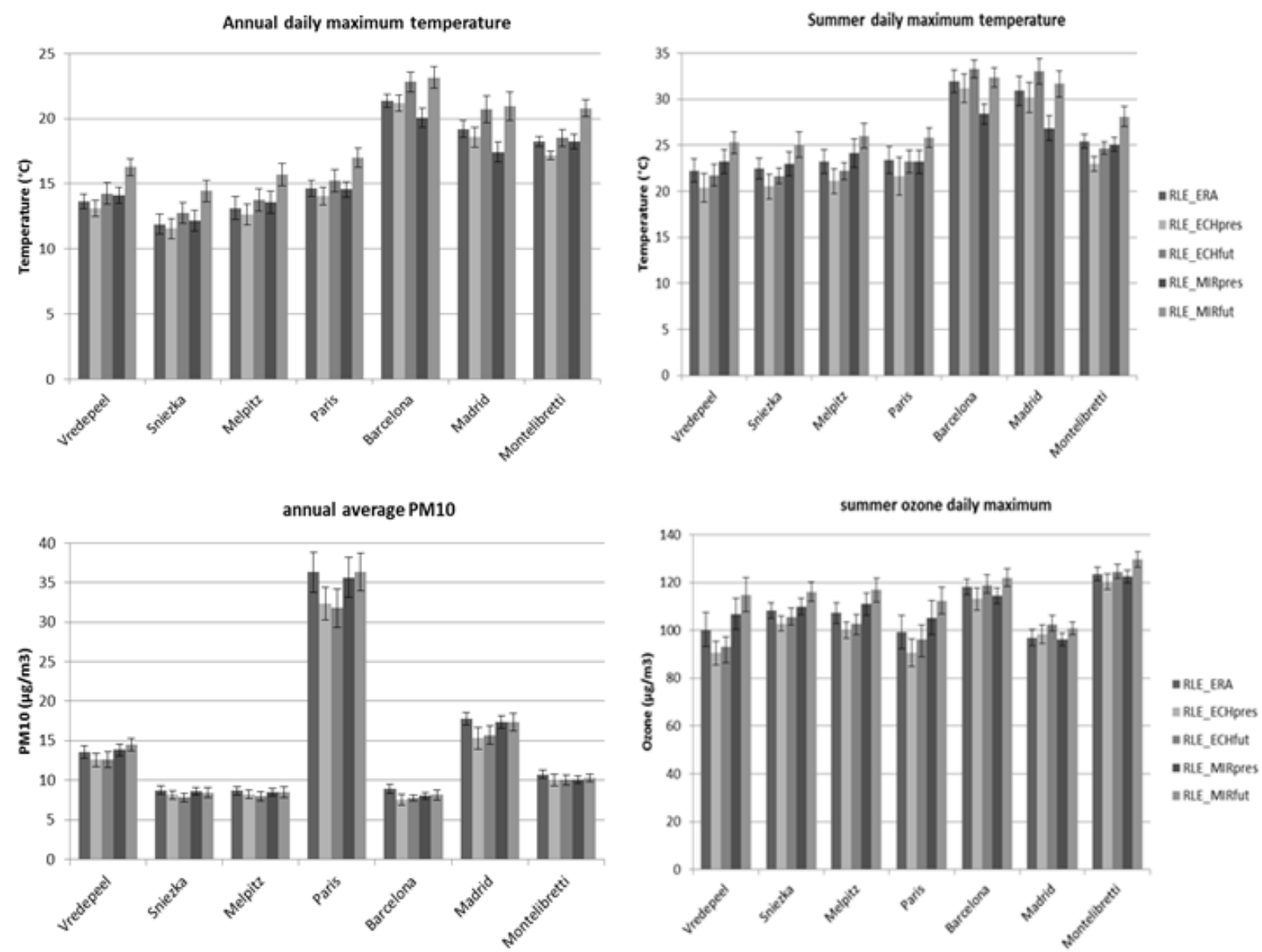

Figure S1. Average values for daily maximum temperature (annual and summer averages) and PM10 (annual) and ozone concentrations (summer averages), with standard deviations to indicate the interannual variability. 
